\title{
Bacterial biofilms in patients with chronic rhinosinusitis: a confocal scanning laser microscopy study*
}

\author{
'Department of Otorhinolaryngology, Akershus University Hospital, Norway \\ ${ }^{2}$ Department of Otorhinolaryngology, Østfold Regional Hospital, Østfold, Norway \\ ${ }^{3}$ University of Oslo, Norway \\ ${ }^{4}$ Drøbak ENT, Drøbak, Akershus, Norway \\ ${ }^{5}$ Department of Pathology, Akershus University Hospital, Norway
}

Kjell Arild Danielsen ${ }^{1,2,3}, \varnothing y s t e i n$ Eskeland ${ }^{1,3,4}$, Katrin Fridrich-Aas', Vivian Cecilie Orszagh ${ }^{5}$, Gregor Bachmann-Harildstad ${ }^{1,3}$, Espen Burum-Auensen ${ }^{1,3}$

Rhinology 52: 150-155, 2014

DOI:10.4193/Rhino13.053

*Received for publication:

May 6, 2013

Accepted: November 6, 2013

\begin{abstract}
Background: Recent research into the pathophysiology of chronic rhinosinusitis suggests an important role for biofilms. They can be detected in both healthy and diseased nasal mucosa. Several different methods of detecting biofilms have been described. This study investigates the presence of biofilm in a larger group of patients with chronic rhinosinusitis undergoing primary functional endoscopic surgery.
\end{abstract}

Methods: Sixty-one patients with chronic rhinosinusitis and 25 controls, with septal deviation, were included from 2010 to 2012. Endonasal biopsies were harvested during surgery, snap frozen in isopentane, cooled on dry ice and stored at $-80^{\circ} \mathrm{C}$. The samples were prepared with Invitrogens' BacLight LiveDead kit, and investigaed with confocal scanning laser microscopy for the presence of biofilm.

Results: In the chronic rhinosinusitis group 55/61 were biofilm positive as opposed to 14/25 in the control group. The difference was highly significant. The odds ratio was 7.2.

Conclusion: Patients with chronic rhinosinusitis have a highly significant increased point prevalence of biofilms compared to controls.

Key words: biofilm, chronic rhinosinusitis, confocal microscopy, FESS

\section{Introduction}

Chronic rhinosinusitis (CRS) is increasingly viewed as an entity composed of several different subgroups of diseases. However, the pathogenesis of CRS is still poorly understood, resulting in a lack of targeted therapy for these patients ${ }^{(1-3)}$.

Recently, several authors reported the presence of bacterial biofilm in the sinonasal mucosa in a high percentage of patients with CRS as opposed to healthy controls, implicating biofilms in the development of CRS ${ }^{(4-6)}$. Biofilms in humans are recognized as the culprit behind a diverse array of chronic infectious diseases, including infectious endocarditis, osteomyelitis and dental caries ${ }^{(7-11)}$.
Biofilms consists of bacterial colonies, often several species, but may also consist of fungi ${ }^{(12)}$. The colonies are protected by an extracellular polymeric substance (EPS), consisting mainly of carbohydrates and proteins, which in large colonies are responsible for its slimy appearance ${ }^{(13)}$.

Bacteria within the biofilm establish gradients for nutrients and oxygen, and communicate by secreting peptides, a process called quorum sensing ${ }^{(14)}$. Bacteria also exchange DNA, notably resistance factors to different antibiotics. These factors contribute to the resilience of biofilms, making them hard to eradicate (15). 
Despite the possible role of biofilms in CRS, some authors do not find significant differences between patients with CRS and controls. Indeed one group, Mladina et al. ${ }^{(16,17)}$, concludes that the reported biofilms represent a mucociliary blanket.

The question arises whether these reported differences could be caused by methodological differences amongst the groups investigating biofilms and CRS? Currently used methods to investigate biofilms in the sinonasal mucosa range from traditional light microscopy, electron microscopy to confocal laser microscopy ${ }^{(6,16-18)}$.

Ha et al. ${ }^{(19)}$ showed that confocal microscopy is better suited for biofilm detection than the other available modalities, and is therefore the method we have chosen in this study.

The primary goal of the present study was to determine the point prevalence of bacterial biofilms in our patients with CRS, after establishing a suitable methodology in a pilot study.

\section{Materials and methods}

A cross-sectional study from 2010 to 2012 was performed in Akershus University Hospital, Norway. Eighty-six consecutive patients undergoing endonasal surgery were included. Sixtyone patients with CRS undergoing functional endoscopic sinus surgery (FESS) as the experimental group, and 25 patients undergoing septoplasty without CRS as controls. The study was approved by the hospital science board and the Regional Ethics Committee (reference number 2009/1720b).

Inclusion and exclusion criteria are detailed in Table 1. Patients were diagnosed with CRS as defined by the EPOS group in 2007
(20). Medical and surgical history, specifically regarding the use of nasal and systemic steroids, smoking, asthma and allergy were obtained. Written consent was obtained from all patients. The study was performed according to the Helsinki declaration.

\section{Tissue collection and preparation}

In the CRS group, tissue samples were harvested from the anterior part of concha media, the uncinate process or the ethmoid bulla during primary sinus surgery. Mucosal samples in the control group were obtained from the anterior part of concha media. Tissue samples were immediately put on moist gauze and transported to the pathology department in clean plastic containers on ice separated by location. Bony tissue was removed and the sample was washed three times in millipore (MQ) water to remove any planktonic bacteria. Each sample was then snap frozen (isopentane cooled with dry ice) in a $1.8 \mathrm{ml}$ cryotube and stored at $-80^{\circ} \mathrm{C}$ ).

At the day of laboratory analysis, samples were thawed at room temperature and washed three times in $25 \mathrm{ml} \mathrm{MQ}$ water ( 0.22 micrometre filter, EMD Millipore Corporation, Billerica, MA, USA) for 60 seconds. They were then transferred to Eppendorf tubes (Eppendorf AG, Hamburg, Germany) containing $1 \mathrm{ml} \mathrm{MQ}$ water. 1,5 $\mu$ l component A (1.67 mM SYTO 9 nucleic acid stain, $1.67 \mathrm{mM}$ propidium iodide solution in DMSO) and $1,5 \mu \mathrm{l}$ component $\mathrm{B}$ (1.67 mM SYTO ${ }^{\otimes} 9$ nucleic acid stain, 18.3 mM propidium iodide solution (Invitrogen`s LIVE/DEAD ${ }^{\circledR}$ BacLight $^{\mathrm{TM}}$ Bacterial Viability Kit, Invitrogen, Burlington, Canada) followed by 10 seconds of stirring (IKA MS3 Digital, VWR International, Radnor, PA, USA). The reagents were incubated for 15 minutes in darkness on a

Table 1. Inclusion and exclusion criteria.

\begin{tabular}{|c|c|c|c|}
\hline CRS group included & CRS Group excluded & Controls included & Controls excluded \\
\hline Primary FESS & Pregnant & $\begin{array}{l}\text { Septal deviation and/or concha } \\
\text { media bullosa requiring surgery }\end{array}$ & $\begin{array}{l}\text { Same as CRS group plus } \\
\text { septal perforation }\end{array}$ \\
\hline Bilateral disease & Immunodeficiency & Above 18 years old & \\
\hline \multirow[t]{8}{*}{ Above 18 years old } & Reduced mucociliary clearance (eg Kartagener) & Lund-Mackay CT score of zero & \\
\hline & Antibiotics within two weeks of operation & & \\
\hline & $\begin{array}{l}\text { Non-invasive fungal balls or invasive fungal } \\
\text { disease }\end{array}$ & & \\
\hline & Systemic vasculitis & & \\
\hline & Granulomatous disease & & \\
\hline & Cocaine abuse & & \\
\hline & Neoplasm & & \\
\hline & Aspirin exacerbated respiratory disease & & \\
\hline
\end{tabular}




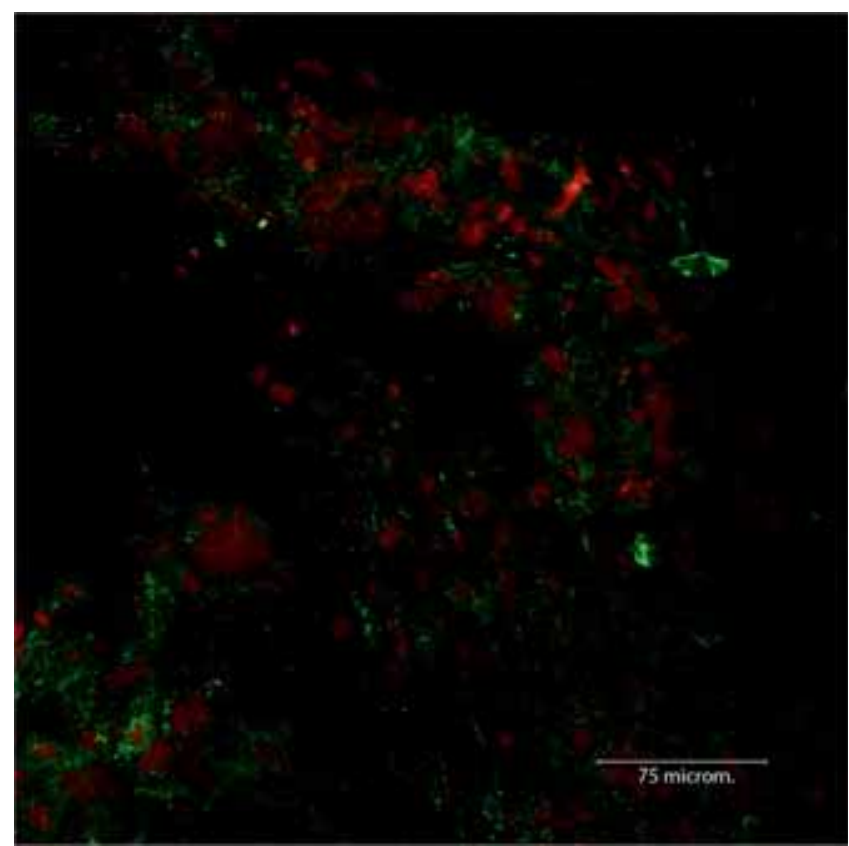

Figure 1. Biofilm positive ( $x 40$ objective). Epithelial cells are the large red structures, and the bacteria are shown as the small green dots dominating the view.

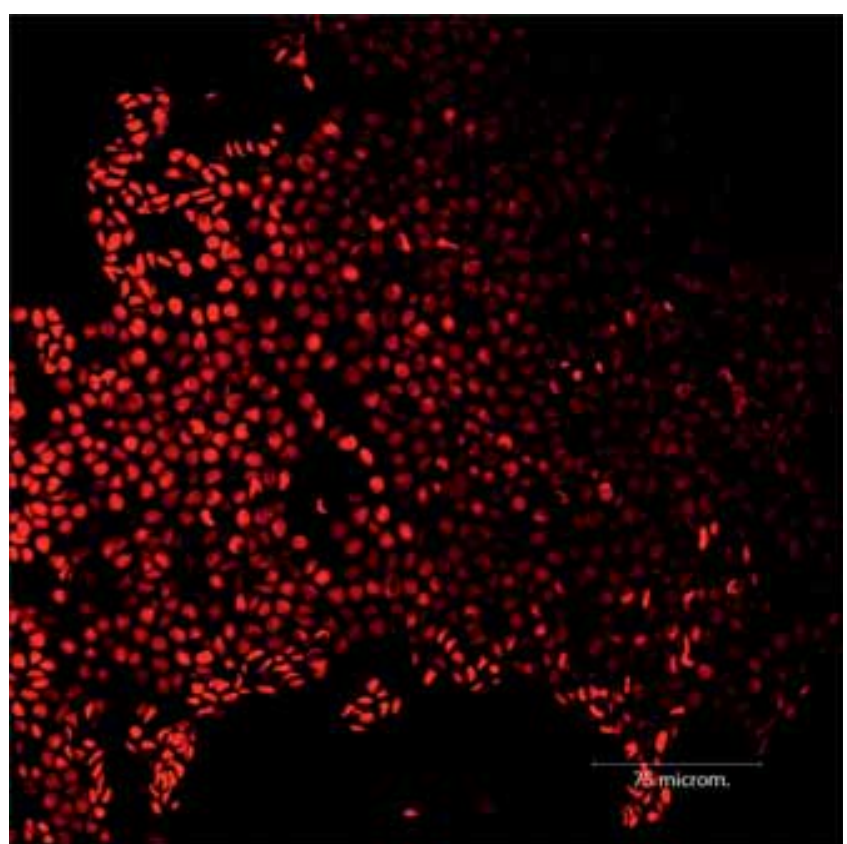

Figure 2. Biofilm negative (x40 objective). Only epithelial cells are seen with no signs of bacteria. nutating mixer (VWR International).

After incubation the samples were washed three times in $25 \mathrm{ml}$ $\mathrm{MQ}$ water. Prior to mounting the tissue biopsies were squeezed gently between two microscope slides (ELKA, Karl Hecht GmbH \& Co KG „Assistent", Sondheim / Rhön, Germany). They were then mounted on a microscope slide with a drop of mounting oil (component C, Invitrogen`s LIVE/DEAD ${ }^{\circledR}$ BacLight $^{\mathrm{TM}}$ Bacterial Viability Kit) and fitted with a cover slip (20x20 mm, $12 \mathrm{~mm}$ thick, VWR International).

After two to three days, visible decomposition had started and after one week the samples were rendered unusable. The fluorescent dyes where stable up to one week after staining, thus tissue decomposition was the limiting factor (unpublished data). We stored the samples in a dark room at $-20^{\circ} \mathrm{C}$. Microscopy was therefore performed on the same day, or the day after, the tissue samples were prepared. We would like to point out that any bacterial growth occurring after preparation of the tissue will not be visible in the confocal microscopy, as only fluorescently marked tissue will light up.

\section{Confocal Laser Microscopy}

Confocal scanning laser microscopy was performed with an upright Leica TCS SP2 AOBS (Leica Microsystems, Wetzlar, Germany). Oil immersion lenses $x 40$ and $x 63$ were used. The examiner was blinded to patient identity and disease status. First the samples were scanned in the fluorescence mode, and then switched to confocal mode. Z-stacks were obtained in areas with possible biofilm. For the confocal part, the Argon laser 488 $\mathrm{nm}$ laser line was used. The strength was set to $15 \%$ and PMT1 was set to 554, PMT2 was set to 517. The strength and reception settings depend on the age of the laser and the thickness of the biopsy. Biofilms were scored when clusters of bacteria with intact membranes, green colour, were present in both the $x-y$ and $x-z$ axes. Typical size of a single bacterium is 0,2 to 2 microns in diameter for spherical species, and 1 to 10 microns in length for nonspherical species. Figure 1 shows a biofilm positive sample, while Figure 2 shows a biofilm negative sample. Z-stacks were useful to form an idea of the three dimensional properties of the biofilms (Figure 3). With samples that the first author found ambiguous regarding the presence of biofilm, the whole group was consulted.

\section{Statistical analyses}

The data obtained in this study were analysed with SPSS 19 (IBM Corp. SPSS, Armonk, NY, USA). Chi-square with continuity correction and odds ratio, when comparing dichotomous data, and student t-test for continuous data were used. For all results a two-sided significance level of $5 \%$ and $95 \%$ confidence interval were used.

\section{Results}

The total number of patients in the CRS group was 61, 23 females and 38 males, and median age was 40 years. There were 25 individuals in the control group, 8 females and 17 males with a 


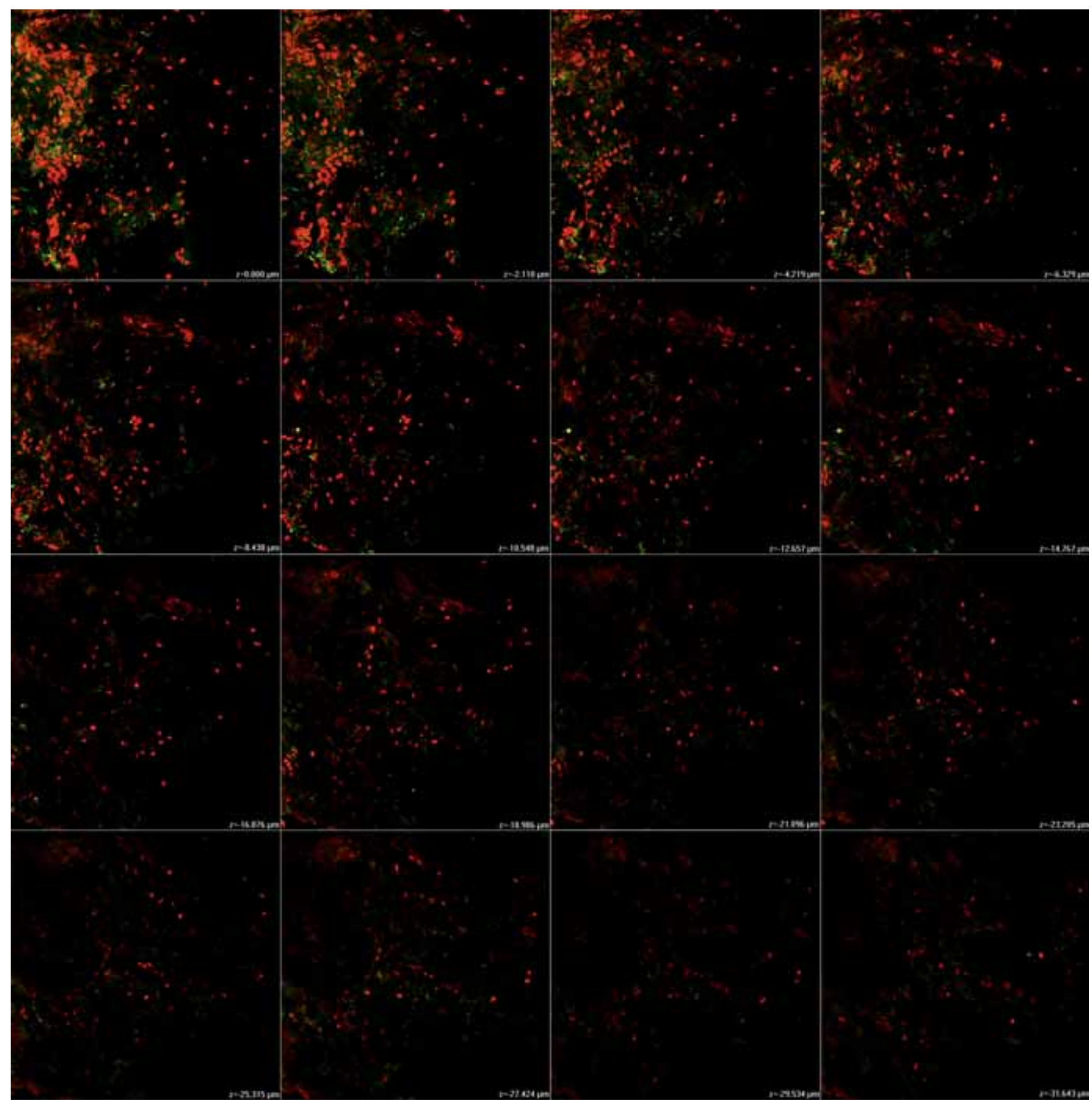

Figure 3. Individual slides in a z-stack, providing a 3-dimensional overview of the sample.

median age of 40 years.

Bacterial biofilms were detected in $90 \%$ of patients with CRS, significantly more prevalent than controls, in which $56 \%$ were biofilm positive ( $p<0.001$, chi-square score 13.1) (Table 2 ).

Patients within the CRS group had significantly higher occurrences of allergy $(50,9 \%$ vs. $17,4 \%, p=0.006)$ and asthma $(34.0 \%$ vs. $8.7 \%, p=0.022$ ) than the controls. In our material, there was a higher rate of current smokers in the control group $(34,0 \%$ vs. 8.7\%, $\mathrm{p}=0.014$ ) (Table 2).

\section{Discussion}

In this study a high point prevalence of biofilms in patients with CRS (90.2\%) was found. This is higher than previously reported, but in line with other studies ${ }^{(4,18,21)}$. A relatively high point prevalence of biofilms was also found in controls (56.0\%), which is higher than most other studies but on level with Bezerra et al. (21). A possible explanation is that patients undergoing septoplasty were used as controls in both this and Bezerra's study. Several other studies reporting low prevalence of biofilms in controls used subjects completely free of nasal complaints undergoing 
Table 2. Results.

$\begin{array}{ccccc} & \text { Controls } & \text { CRS Group } & \text { p-value } & \\ \text { No. of patients } & 25 & 40 & 38 & \\ \text { Age; Median } & 40 & 23 & (p=0.014) \\ \text { Male } & 17 & 11.1 \% & (p=0.006) \\ \text { Female } & 8 & 50.9 \% & (p=0.022) & (p<0.001)\end{array}$

skull base surgery ${ }^{(4,6)}$. This may point to a role for biofilms in the pathogenesis of patients with nasal obstruction without CRS. Further studies are needed.

In the current study, we have harvested biopsies from the structures surrounding the middle meatus as not all patients underwent surgery of the frontal recess, posterior ethmoid cells or sphenoid sinus. In a later study, we plan to investigate whether there is an unequal distribution of biofilms in the different compartments of the sinonasal cavity.

We chose to follow the protocol for biofilm detection with confocal microscopy outlined by Psaltis et al. ${ }^{\left({ }^{6}\right)}$ with cryopreservation instead of direct microscopy for convenience. In a later article, Foreman et al. ${ }^{(4)}$ reported that cryopreservation gave results similar to direct microscopy.

Research into the pathophysiology of CRS increasingly points out the need to differentiate several distinct subgroups; e.g. Samters triad, ciliary dysfunction, eosinophilia and immunodeficiency ${ }^{(1)}$. In this study, we wanted to examine the association with biofilm infection in patients with CRS without these underlying conditions at the time of primary rhinological surgery. In our opinion, a biofilm point prevalence of above $90 \%$ in this group strongly suggests such a connection.

To date three main modalities are used to investigate CRS and biofilms: Confocal, electron and light microscopy ${ }^{(4,6,16-18)}$. Light microscopy, which is cheap and readily accessible, has the drawbacks of low resolution and inability to visualize the individual bacteria, making it hard to differentiate between biofilms and planktonic bacteria. Scanning electron microscopy offers great resolution, but is expensive and labour intensive. It is also difficult to distinguish dead bacteria in the mucociliary blanket from actual biofilms, which are bound irreversibly to the epithelial cells. We therefore speculates that this is the reason why Mladina et al. ${ }^{(16,17)}$ found biofilms in almost all subjects, both patients and controls.

Ha et al. ${ }^{(19)}$ concludes that confocal microscopy is best suited for detection of biofilms in the sinonasal mucosa. Confocal microscopy features the ability to distinguish between bacteria with intact cell membranes at the time of preparation, indicating vital bacteria, and dead bacteria with compromised cell membranes. Additionally, it provides the opportunity to scroll into the depth of the tissue sample, the z-axis, and allows an insight into the three dimensional properties of a suspected biofilm. On the downside, confocal scanning laser microscopy is expensive and labour intensive, and also not widely available.

In conclusion, this study strengthens the mounting evidence of the role of biofilms in patients with CRS. The control group with nasal congestion without CRS had $56 \%$ biofilm positivity, indicating a role for biofilms in this group as well. Furthermore, the methodology of biofilm research in CRS should become more standardized in the future, and we suggests the merits of confocal microscopy.

Further studies are needed in this area, and we plan to investigate the role of biofilms in the setting of nasal polyps, inflammation associated with biofilm colonies and postoperative results between biofilm positive and negative subjects.

\section{Acknowlegdement}

This study has been supported by means and facilities from Akershus University Hospital, Norway and by the University of Oslo. 


\section{Authorship contribution}

KAD: Planned and implemented the study. Performed microscopy on all the samples. Analysed data and drafted the manuscript. Final approval of version to be submitted. $\varnothing \mathrm{E}:$ Planned and implemented the study. Participated in microscopy, and was responsible for the entire main database. Analysed data and reviewed the manuscript. Final approval of version to be submitted.

KFA: Planned and implemented the study. Handled the samples, anonymised and stored them. Reviewed scoring on difficult samples. Reviewed the manuscript. Final approval of version to be submitted.

VCO: Planned and implemented the study. Handled the samples, anonymised and stored them. Reviewed scoring on difficult samples. Reviewed the manuscript. Final approval of version to be submitted.

GBH: Planned and implemented the study. Helped with logistics concerning the biopsies in the operating theatre. Reviewed the manuscript. Final approval of version to be submitted.

EBA: Planned and implemented the study. Responsible for establishing the protocol of biopsy-staining. Analysed data and reviewed the manuscript. Final approval of version to be submitted.

\section{Conflicts of Interest}

None of the authors report any conflicting interests.

\section{References}

1. Fokkens WJ, Lund VJ, Mullol J, Bachert C, Alobid I, Baroody F, et al. European Position Paper on Rhinosinusitis and Nasal Polyps 2012. Rhinol Suppl. 2012; (23): 3-298.

2. Bachert C, Van BN, Toskala E, Zhang N, Olze $\mathrm{H}$, Scadding $\mathrm{G}$, et al. Important research questions in allergy and related diseases: 3-chronic rhinosinusitis and nasal polyposis - a GALEN study. Allergy. 2009; 64: 520-533.

3. Ooi EH, Wormald PJ, Tan LW. Innate immunity in the paranasal sinuses: a review of nasal host defenses. Am J Rhinol. 2008; 22: 13-19.

4. Foreman A, Psaltis AJ, Tan LW, Wormald PJ. Characterization of bacterial and fungal biofilms in chronic rhinosinusitis. Am J Rhinol Allergy. 2009; 23: 556-561

5. Li H, Wang D, Sun X, Hu L, Yu H, Wang J. Relationship between bacterial biofilm and clinical features of patients with chronic rhinosinusitis. Eur Arch Otorhinolaryngol. 2012; 269: 155-163.

6. Psaltis AJ, Ha KR, Beule AG, Tan LW, Wormald PJ. Confocal scanning laser microscopy evidence of biofilms in patients with chronic rhinosinusitis. Laryngoscope. 2007; 117 1302-1306

7. Donlan RM, Costerton JW. Biofilms: survival mechanisms of clinically relevant microorganisms. Clin Microbiol Rev. 2002; 15: 167193.

8. Marsh PD, Bradshaw DJ. Dental plaque as a biofilm. J Ind Microbiol. 1995; 15: 169-175.
9. Simoes M. Antimicrobial strategies effective against infectious bacterial biofilms. Curr Med Chem. 2011; 18: 2129-2145.

10. Smith A, Buchinsky FJ, Post JC. Eradicating chronic ear, nose, and throat infections: a systematically conducted literature review of advances in biofilm treatment. Otolaryngol Head Neck Surg. 2011;144(3): 338-347.

11. Keir J, Pedelty L, Swift AC. Biofilms in chronic rhinosinusitis: systematic review and suggestions for future research. J Laryngol Otol. 2011; 125: 331-337.

12. Harriott MM, Noverr MC. Importance of Candida-bacterial polymicrobial biofilms in disease. Trends Microbiol. 2011; 19: 557-563.

13. Hall-Stoodley L, Stoodley P. Evolving concepts in biofilm infections. Cell Microbiol. 2009; 11: 1034-1043.

14. Li YH, Tian X. Quorum sensing and bacterial social interactions in biofilms. Sensors (Basel). 2012; 12: 2519-2538.

15. Al-Mutairi D, Kilty SJ. Bacterial biofilms and the pathophysiology of chronic rhinosinusitis. Curr Opin Allergy Clin Immunol. 2011; 11: 18-23.

16. Mladina R, Skitarelic N, Music S, Ristic M. A biofilm exists on healthy mucosa of the paranasal sinuses: a prospectively performed, blinded, scanning electron microscope study. Clin Otolaryngol. 2010; 35: 104-110.

17. Mladina R, Skitarelic N. Biofilm--the other name for the regular mucosal blanket. Med
Hypotheses. 2010; 75: 391-392.

18. Hochstim CJ, Masood R, Rice DH. Biofilm and persistent inflammation in endoscopic sinus surgery. Otolaryngol Head Neck Surg. 2010; 143: 697-698.

19. Ha KR, Psaltis AJ, Tan L, Wormald PJ. A sheep model for the study of biofilms in rhinosinusitis. Am J Rhinol. 2007; 21: 339-345.

20. Fokkens W, Lund V, Mullol J. European position paper on rhinosinusitis and nasal polyps 2007. Rhinol Suppl. 20: 1-136.

21. Bezerra TF, Padua FG, Gebrim EM, Saldiva $\mathrm{PH}$, Voegels RL. Biofilms in chronic rhinosinusitis with nasal polyps. Otolaryngol Head Neck Surg. 2011; 144: 612-616.

Kjell Arild Danielsen

Øre-nese-halsavdelingen

Sykehuset Østfold HF, Pb 16

1603 Fredrikstad

Østfold

Norway

Tel: $+47-08600$

Fax: +47-6986 0723

Mail: k.a.danielsen@medisin.uio.no 